\title{
Attention in bisensory simultaneous short-term memory ${ }^{1}$
}

\author{
RHEA L. DORNBUSH ${ }^{2}$ \\ NEW YORK MEDICAL COLLEGE
}

\begin{abstract}
Subjects were presented with simultaneously visual and auditory material. They were instructed to attend to only one modality but were required to recall materials from both modalities, one before the other. Results supported a previous suggestion that visual recall is minimum under conditions of simultaneous presentation. That is, in situations where $S$ is allowed to divide his attention between both inputs, visual recall was no greater than when his attention was directed away from visual input and to auditory input. It was noted that this difference in recall between the two modalities was limited to verbal material, as other data indicate the opposite effect for nonverbal material.
\end{abstract}

Previous work on bisensory simultaneous short-term memory employing the visual and auditory modalities has indicated that visual recall is inferior to auditory recall (Broadbent, 1958; Buschke, 1962; Dornbush, 1968a, b; Margrain, 1967). Dornbush (1968b) found visual recall so considerably less than auditory that she suggested that the amount recalled visually was, perhaps, no more than might be expected on an incidental level. Specifically, in an ordinary bisensory situation, i.e., where attention is supposedly divided equally between the two inputs, visual recall was at nearly the identical low level as obtained in a situation where S's attention was drawn away from visual material and to the auditory material (accomplished by shadowing the latter input). That is, recall in both modalities was the same whether $S$ was actively attending to the auditory modality or was at liberty to divide his attention between modalities.

Thus, the present experiment was designed to determine exactly how minimal visual recall was under the bisensory simultaneous condition and when $S$ was not actively involved in another activity, such as shadowing. Specifically, Ss were stimulated bisensorily, instructed to attend to only one modality, but required to recall materials from both modalities.

\section{METHOD}

\section{Apparatus}

Materials ${ }^{3}$ were presented on 16-mm film (24 frames/sec) with synchronous sound track by means of a Kalart projector. The exact auditory sequences intended for pairing with the appropriate visual sequences were recorded in a sound studio by the $\mathrm{E}$; precise control of synchrony was accomplished by splicing the auditory tape. That is, speech recordings were spliced into the film so that each utterance of a stimulus item commenced within the first half frame of its associated visual stimulus $( \pm 21 \mathrm{msec})$.

Visual stimuli were made by "Letraset" instant black letters exposed singly on white $81 / 2 \times 11 \mathrm{in}$. cards, which were then filmed and appropriately edited. The contrast ratio, as displayed, was approximately $80 \%$. The interstimulus and interlist intervals for visually presented materials consisted of clear leader that was slightly less bright than the stimulus sequence, thus minimizing the negative afterimage. While an attempt was made to equate subjectively the auditory and visual intensities, it seemed reasonable to assume that the intensities might not be the crucial variable as long as the material was clearly and easily discriminable (Sperling, 1963).

\section{List Preparation and Presentation}

The first 10 letters of the alphabet, $A$ through $J$, and the digits 0 through 9 were employed. These letters were chosen as pilot studies measuring tachistoscopic thresholds of the 26 letters of the alphabet indicating that each item was recognized with equal facility. As material was not the main concern of the present experiment and had been investigated previously (Dornbush, 1968a, b), data were not analyzed with respect to material combinations. However, it should be noted that there were no split lists. That is, any given list was either all numbers or all letters.

Each list contained six items. Altogether, 48 lists were required to produce: 24 pairs of lists sampling all possible combinations of three rates of presentation-fast (F), medium (M), slow (S); two orders of report-first, second; two levels of attention-attend auditory, attend visual; and two modalities-auditory, visual.

For half of the lists, Ss were required to attend to the auditory modality; for half of these lists, Ss were to report the attended modality first and then the lists in the unattended modality.

Pairs of lists at each rate were presented in a block in the medium, slow, fast order. That is, order of report and modality attended to were counterbalanced in a prearranged random order within each rate of presentation.

Visual items were presented for $.5 \mathrm{sec}$ (12 frames); the duration of the auditory items averaged 10 frames, or $.42 \mathrm{sec}$. Materials were presented at three rates: (1) $1 \mathrm{pair} / .5 \mathrm{sec}$, no interstimulus interval (fast); (2) $1 \mathrm{pair} / \mathrm{sec}, .5$-sec interstimulus interval (medium); (3) 1 pair $/ 2 \mathrm{sec}, 1.5$-sec interstimulus interval (slow). There was a $20-\mathrm{sec}$ interlist interval, with $15 \mathrm{sec}$ for recall and a ready signal given $.5 \mathrm{sec}$ before the start of each list.

\section{Subjects}

Sixty undergraduates in psychology, satisfying a course requirement, served as Ss. Each $S$ received every condition, i.e., the experiment was set up as a total within-S design.

\section{Administration}

The Ss were run as a group in a large auditorium with a projection booth. They were centrally seated so that no $S$ viewed the screen at an extreme angle. All Ss were given general short-term memory instructions, with emphasis placed on the necessity of correct serial order of items in written response. Prior to the presentation of each list, Ss were told which channel to attend to; this was indicated by the word "auditory" or "visual" given after the ready signal. Immediately after the presentation of the last item in each list, the word "auditory" or "visual" was again spoken, indicating the prescribed order of report.

Each $S$ was given answer sheets in booklet form-each page represented one rate-with space provided for each response, auditory items were on one half of the page, visual items on the other half. Half of the Ss received answer sheets with visual on the right side, auditory on the left; the other half received the reverse order.

\section{RESULTS}

The data for each $S$ for each sequence of six items were scored according to the accuracy of responses both in nature and in location, i.e., on an item-by-item basis permitting individual scores to take on values between 0 to 6 . The average scores 


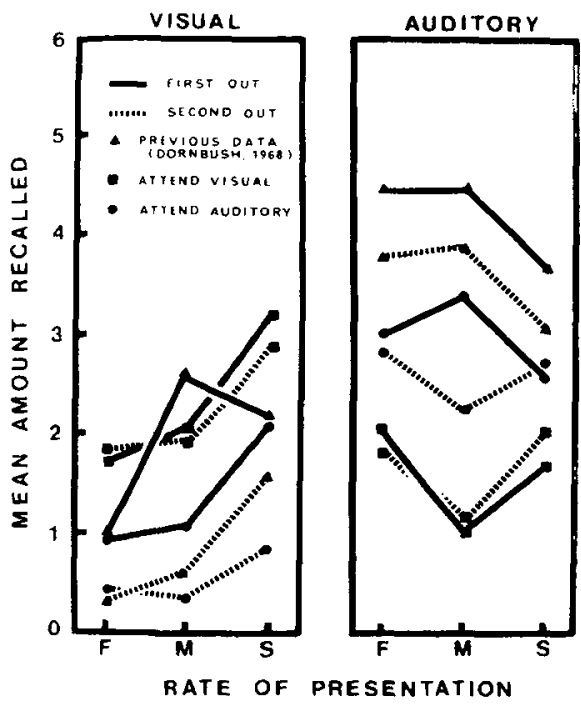

1. Average scores for each task compared with previously collected data from a typical bisensory situation (Dornbush, 1968b).

for each task are shown in Fig. 1. They are compared with previously collected data from an ordinary bisensory situation (Dornbush, 1968b). Inasmuch as this latter situation yields data that are highly stable and reliable even under very different laboratory and procedural conditions (Dornbush, 1968a, b; Margrain, 1967), it was not considered necessary to nu such a group again. That is, the main effects of modality, order, and rate yield similar functions and similar differences in means in every experimental condition studied.

As seen in Table 1, three of the four main effects were significant: More auditory material was recalled than visual; the channel recalled first was recalled best; and recall was greatest at the slowest rate. A Scheffé test indicated that the medium rate differed significantly from the slow rate $[F(2,59)=3.37, \mathrm{p}<.05]$.

As higher-order interactions are often difficult to interpret, and even more often are meaningless, four three-way analyses of variance were undertaken. Perhaps the most relevant interaction in terms of the present experiment was Attention by Modality $[F(1,59)=15.87, p<.001]$. This interaction indicated that while more was recalled in the attended modality, the difference in the amount recalled in the two modalities was considerably greater when $S$ was attending to the auditory input. Specifically, when $S$ was attending to the auditory input, auditory recall was 2.83 , and visual recall was 0.96 , as might be expected; on the other hand, when attending to visual input, visual recall was 2.27 , and auditory recall was as great as 1.64 .

In addition to the Attention by Modality interaction, three other two-way interactions were significant. These were: Modality by Order $[F(1,59)=4.52$, $\mathrm{p}<.05]$, with material recalled first being superior to material recalled second but with the disparity greater in the visual channel than it was in the auditory channe 1; Modality by Rate $\{F(2,118)=15.01, p<.001\}$, with visual recall least at the fastest rate and auditory recall least at the medium rate (in this interaction, visual results are similar to those typically obtained, i.e., increasing recall with decreasing rate; on the other hand, auditory recall is somewhat less consistent, i.e., typically a decreasing amount is recalled with decreasing rate, while, here, the amount decreases and then increases at the slowest rate); Attention by Order $[F(1,59)=13.99, p<.001]$, with considerably more recalled in the channel reported first than in the channel reported second when attention was given to the auditory modality, while there was little difference in the amount recalled in the first and second channels when $S$ was attending to the visual input. The basis for this lack of difference in recall between first and second channel can be more readily understood by examining the nonsignificant Attention by Modality by Order interaction, with particular attention to the attend-visual conditions. In this condition, recall in a given modality was approximately the same whether the modality was recalled first or second; thus, when scores were collapsed across modalities for the Attention by Order interaction, the scores balanced each other. More specifically, auditory recall in the first position was 1.61 , visual recall in the first position was 2.33 , auditory recall in the second position was 1.66 , and visual recall in this position was 2.21 .

Of the triple interactions, the Attention by Modality by Rate interaction was

Table 1

Means and (SDs) for Main Effects of Attention, Modality, Order of Report and Rate of Presentation

\begin{tabular}{|c|c|c|c|c|c|c|c|c|}
\hline Task & & & & & & & DF & $\mathbf{P}$ \\
\hline $\begin{array}{l}\text { Attention: } \\
\text { Modality: } \\
\text { Order: } \\
\text { Rate: }\end{array}$ & $\begin{array}{l}\text { Aud. } \\
\text { Aud. } \\
\text { Fst. } \\
\text { Fast }\end{array}$ & $\begin{array}{l}1.89 \\
2.23 \\
2.08 \\
1.85\end{array}$ & $\begin{array}{l}(.66) \\
(.83) \\
(.67) \\
(.68)\end{array}$ & $\begin{array}{l}\text { Vis. } \\
\text { Vis. } \\
\text { Sec. } \\
\text { Med. } \\
\text { Slow }\end{array}$ & $\begin{array}{l}1.95 \\
1.61 \\
1.77 \\
1.56 \\
2.36\end{array}$ & $\begin{array}{l}(.76) \\
(.70) \\
(.80) \\
(.68) \\
(1.07)\end{array}$ & $\begin{array}{l}1,59 \\
1,59 \\
1,59 \\
2,118\end{array}$ & $\begin{array}{l}\text { NS } \\
<.001 \\
<.001 \\
<.001\end{array}$ \\
\hline
\end{tabular}

significant $[F(2,118)=3.73, p<.05]$, reflecting mainly the differences in the auditory recall scores that, as mentioned previously, were not as regular as visual data. Thus, while visual recall increases with decreasing rate regardless of the attended modality, auditory recall is greatest at the fastest rate when $S$ attends the visual modality and greatest at the slowest rate when attending the auditory modality.

\section{DISCUSSION}

The impetus for this study was the suggestion that visual recall in the typical bisensory condition is minimal. This, in fact, appears to be very nearly the case. Examination of Fig. 1 indicates similar visual scores for typical bisensory and attend auditory conditions (except for a single point-the medium rate on the channel recalled first). It should also be noted that the amount recalled visually when $S$ was attending to visual input, which should be the optimal visual condition, was still less than auditory recall when $S$ attended to both visual and auditory inputs. Additionally, auditory recall, when $S$ attended to the visual modality, is high relative to visual input when $S$ attended to the auditory modality, as mentioned previously.

Margrain (1967), investigating the short-term memory as a function of input modality by directing S's attention to a given modality (via shadowing), obtained functionally identical results as the above and very nearly identical results when Ss were required to write material on one channel as it occurred. Margrain concluded, as does the present author, "that input modality appears to be far more important for recall than was directing S's attention to a list during input when that list might or might not have been subsequently required for recall [p. 109]."

However, why the differences in recall in the two modalities exist is still not understood. But there is evidence that these differences are limited to verbal material. That is, Lindsay, Cuddy, and Tulving (1965) and Tulving and Lindsay (1967) performed a similar attention-directing experiment with nonverbal simultaneously presented visual and auditory stimuli. Ss were presented with both stimuli and toid to identify both but to concentrate on and report one stimulus first; and, in an identical condition to the present experiment, $S$ was presented with stimuli from both modalities simultaneously and was required to attend and judge only one of the modalities. Contrary to the present findings, Tulving et al found performance measured in information transmitted in 
bits, greater for visual stimuli than for auditory stimuli. Again, these data were obtained with nonverbal stimuli, and, apparently, the verbal or nonverbal nature of the task is a highly relevant determiner of results (Milner, 1960). Milner, investigating laterality effects, states: "We have to keep in mind the nature of the stimulus material which the nervous system must handle; this means not only the sensory channel, but also the verbal or nonverbal nature of the information being processed [p. 194]."

Obviously, then, further detailed investigation of modality differences as a function of material is necessary. The work on cerebral dominance and temporal lobe lesions seems the most promising source of this information at this time.

\section{REFERENCES}

BROADBENT, D. E. Perception and communication. London: Pergamon Press, 1958.

BUSCHKE, $\mathrm{H}$. Auditory and visual interaction in immediate memory. Psychiatric Research, 1962, 1, 229-237.

DORNBUSH, R. L. Input variables in bisensory memory. Perception \& Psychophysics, 1968a, 4, 41-44.

DORNBUSH, R. L. Shadowing in bisensory memory. Quarterly Journal of Experimental Psychology, 1968b, 20, 225-231.

LINDSAY, P. H., CUDDY, L., \& TULVING, E. Absolute judgments of simultaneously presented visual and auditory stimuli. Psychonomic Science, 1965, 2, 211-212.

MARGRAIN, S. A. Short-term memory as a function of input modality. Quarterly Joumal of Experimental Psychology, 1967, 19, 109-114.

MILNER, B. Laterality effects in audition. In V. B. Mountcastle (Ed.), Interhemispheric relations and cerebral dominance. Baltimore: Johns Hopkins Press, 1962.

SPERLING, G. A model for visual memory tasks. Human Factors, 1963, 5, 19-31.

TULVING, E., \& LINDSAY, P. H. Identification of simultaneously presented visual and auditory stimuli. Acta Psychologica, 1967, 27, 101-109.

\section{NOTES}

1. This research was supported by grants from the National Institute of Mental Health (MH 15600), The American Philosophical Society, and The Society of the Sigma Xi.

2. Address: Department of Psychiatry, New York Medical College, Flower and Fifth Avenue Hospitals, 5 East 102nd Street, New York, New York 10029.

3. A more detailed description of materials is presented elsewhere (Dombush, 1968a, b).

(Accepted for publication July 7, 1969.) 